\title{
Desempenho produtivo de quatro linhagens de frangos de corte
}

\author{
Productive performance of four cut chickens lineages
}

\author{
Daniel Noal Moro $^{1}$ Irineu Zanella ${ }^{2}$ Élsio Antônio Pereira de Figueiredo ${ }^{3}$ \\ José Henrique Souza da Silva ${ }^{4}$
}

\section{RESUMO}

Estipulou-se como objetivos experimentais avaliar os parâmetros produtivos das linhagens da EMBRAPA $e$ compará-los a duas marcas comerciais no período de 1-56 dias, o trabalho experimental foi realizado no Centro Nacional de Pesquisas em Suínos e Aves, da EMBRAPA, localizado na cidade de Concórdia, Santa Catarina, no período de 06 de julho de 2001 a 30 de agosto de 2001, totalizando 56 dias de experimento. Cada box possuía 1,65m X 1,75m, a lotação inicial era de 40 animais por box $\left(13,88\right.$ frangos $\left.\mathrm{m}^{-2}\right)$, separados por sexo e linhagem. Foram utilizados 48 boxes, sendo 12 de cada linhagem e seis de cada sexo, totalizando 1.920 animais alojados. Os animais receberam dietas formuladas de acordo com o NRC (1994). Foram estimados os seguintes parâmetros: consumo de ração, peso médio, ganho médio diário de peso, conversão alimentar e índice de eficiência produtiva. $O$ delineamento experimental foi o Inteiramente Casualizado, com esquema fatorial (4x2), não havendo interação para nenhum dos fatores estudados. Foi realizada uma análise de variância sobre os dados obtidos $e$ aplicado o teste de Tukey quando o " $F$ " foi significativo. Concluiu-se que as linhagens da EMBRAPA possuem boas características zootécnicas, seu desempenho zootécnico ficou próximo ao que é indicado no manual das linhagens de frangos de corte comerciais.

Palavras-chave: linhagem, sexo, frango, avaliação.

\section{ABSTRACT}

The present research aims at evaluating the productive parameters of the EMBRAPA lineages and comparing them to two commercial breeds in the period of 1 to 56 days. The experiment was done in the National Center for Poultry and Swine Research, at EMBRAPA, located in Concordia, Santa Catarina, Brazil. The research was carried out from July 6, 2001 to Aug 30, 2001, in a 56-day-experiment. Each box had $1.65 \times 1.75 \mathrm{~m}$. The initial population was of 40 animals per box (13.88 broilers $\left.m^{-2}\right)$, separated by sex and lineage. Forty-eight boxes were used, being 12 of each lineage and 6 of each sex containing 1920 animals in total. The animals received diets formulated according to the NRC (1994). The following parameters were estimated: food consumption, average weight, daily average weight gain, feeding conversion, and productive efficiency index. The experimental outline was the Entirely Randomized one with factorial scheme (4x2), having no interaction with any of the factors studied. A variance analysis over the obtained data was performed and the Tukey test was used when the " $F$ " was significant. It was concluded that the EMBRAPA lineages have good husbandry characteristics. Its performance was close to what it is indicated in the commercial broiler manual.

Key words: lineage, sex, broiler, evaluation.

\section{INTRODUÇÃO}

A carência de proteína animal, em grande parte da população mundial, tem forçado um estímulo cada vez maior às especializações e à adoção de determinadas tecnologias, no sentido de se conseguir uma máxima produção, com menor custo, em menor tempo, na menor área possível. Diante desse contexto, a avicultura engloba-se

Mestrando em Zootecnia, Universidade Federal de Santa Maria (UFSM), RS, Brasil, Rua José Mariano da Rocha, 165, Bairro N.S.Lourdes, 97060-180, Santa Maria, RS, Brasil. E-mail: dnmoro@bol.com.br. Autor para correspondência.

${ }^{2}$ Orientador, Professor, Doutor, Departamento de Zootecnia, UFSM

${ }^{3}$ Pesquisador PhD, Centro Nacional de Pesquisas em Suínos e Aves (CNPSA), Concórdia, SC, Brasil.

${ }^{4}$ Professor, PhD, Departamento de Zootecnia, UFSM. 
perfeitamente como atividade produtora de proteína animal, sendo as aves uma das espécies zootécnicas mais eficientes em transformar alimentos vegetais em alimentos de alto valor protéico para o homem.

Segundo TALAMINI \& FILHO (2003), a produção de frangos de corte teve um acréscimo anual de $8,34 \%$ nos últimos seis anos, tornando nosso país um dos mais importantes fornecedores de carne para o mundo, bem como ocorreu um aumento no consumo per capita de $1,62 \mathrm{~kg}$ por ano, neste período, atingindo em 2002, $31 \mathrm{~kg}$ por habitante. Apesar de toda a evolução do setor, a avicultura brasileira está na dependência, praticamente total, de material genético proveniente do exterior, tanto em frangos de corte quanto em poedeiras. Esta tem sido uma preocupação dos pesquisadores brasileiros na área de melhoramento animal. Na década de 80, a Granja Guanabara foi adquirida pela EMBRAPA, e nela existia uma linhagem de frangos de corte em desenvolvimento a G-190. De acordo com CAMPOS (2000), dois fatores foram as causas do insucesso desta linhagem: o tamanho reduzido da população e a alta incidência de doenças.

Com a instalação do Centro Nacional de Pesquisa de Suínos e Aves em Concórdia, a EMBRAPA deu continuidade ao programa de melhoramento genético avícola. Apesar do pouco aporte financeiro, duas décadas após, desenvolveu duas linhagens de frangos de corte que estão disponíveis no mercado. Assim, para conquistar o mercado das empresas avícolas brasileiras, necessário se faz avaliar estas linhagens. Também se faz necessário um futuro trabalho de avaliação de matrizes, pois se precisa de um equilíbrio entre o desempenho da matriz de corte e do seu produto, o frango de corte. Diante de tais variáveis, se faz necessária uma maior conscientização dos empresários do setor, da importância em valorizar aquilo que é nosso e não somente aquilo que vem de outros países. Com este objetivo, as duas linhagens desenvolvidas pela EMBRAPA foram avaliadas quanto ao desempenho produtivo de 1 a 56 dias de idade, sendo estas comparadas com duas das principais linhagens de frangos de corte do mercado avícola brasileiro.

\section{MATERIAL E MÉTODOS}

O experimento foi conduzido no CNPSA, localizado na vila Tamanduá, em Concórdia-SC, no período de 06 de julho a 30 de agosto de 2001 , totalizando 56 dias de experimento. O período experimental foi de 56 dias por ser esta a idade média que possibilita melhor eficiência econômica para venda de cortes de frango, tanto no mercado interno como para exportação. O aviário possui 112 boxes, com as seguintes dimensões $1,75 \mathrm{~m}$ x $1,65 \mathrm{~m}$, sendo equipado com um comedouro tipo tubular e um bebedouro pendular para cada box de 40 aves. A taxa de lotação inicial do experimento foi de 13,85 aves por metro quadrado. O piso do aviário é de concreto, sendo utilizado como cama aviária maravalha. Os pintos tiveram o círculo de proteção até os nove dias de idade em função da época do ano (inverno). A orientação solar da construção é leste-oeste, não apresentando ventilador e nebulizador. Os animais receberam dietas formuladas de acordo com o NRC (1994), isonutritivas, tendo recebido quatro dietas, conforme a fase ao longo do período. As matrizes que deram origem aos pintos utilizados tinham 40 semanas de idade.

Os pintos utilizados no experimento são assim descritos: 240 frangos de corte machos da linhagem A; 240 frangos de corte fêmeas da linhagem A; 240 frangos de corte machos da linhagem B;240 frangos de corte fêmeas da linhagem B; 240 frangos de corte machos da linhagem EMBRAPA 1; 240 frangos de corte fêmeas da linhagem EMBRAPA 1; 240 frangos de corte machos da linhagem EMBRAPA 2; 240 frangos de corte fêmeas da linhagem EMBRAPA 2.

Totalizaram 1920 animais no experimento, sendo que cada unidade experimental foi composta de 40 animais, que foram sorteados aleatoriamente, separados por sexo e linhagem. Os animais foram pesados aos 56 dias de idade. Foram estimados os seguintes parâmetros: consumo médio de ração por ave $(\mathrm{CR})$, peso médio (PM), ganho médio diário de peso (GMD), conversão alimentar (CA) e índice de eficiência produtiva (IEP). Este índice é muito utilizado pela indústria para remunerar seus integrados, para cada sexo e para cada idade do lote existe um valor, ele vem aumentando significativamente nos últimos anos devido às melhorias nos mais diferentes segmentos da produção como nutrição, genética, sanidade, manejo, ambiência, entre outros. É obtido pelo peso médio do lote no dia da retirada, multiplicado pela viabilidade criatória que é obtida pela subtração de 100, menos a mortalidade. Todo esse numerador é dividido pela idade do lote na retirada, multiplicado pela conversão alimentar e, após a divisão, esse valor obtido é multiplicado por cem. O IEP foi calculado aos 56 dias de idade das aves.O delineamento experimental foi o 
inteiramente casualizado, com quatro tratamentos, distribuídos num esquema fatorial $4 \times 2$, sendo estudadas quatro linhagens de frango de corte, em que cada linhagem tinha os dois sexos, seis boxes de cada sexo e linhagem, totalizando 48 boxes com 1.920 animais envolvidos no experimento. Os dados foram submetidos à ANOVA e quando o "F" foi significativo, foi aplicado o teste de Tukey para comparar as médias. Todas estas análises foram realizadas com o auxilio do pacote estatístico SAS PROGRAM (1997).

\section{RESULTADOS E DISCUSSÃO}

Não se verificou interação significativa entre linhagem e sexo em nenhum dos parâmetros avaliados. Na tabela 1, é apresentado o desempenho zootécnico das quatro linhagens avaliadas no período de 1-56 dias de idade. O PM não diferiu entre as linhagens da EMBRAPA 1 e Linhagem A $(\mathrm{P}>0,05)$. A EMBRAPA 1 foi superior estatisticamente à EMBRAPA 2 e Linhagem B, porém o PM não diferiu estatisticamente entre a EMBRAPA 2 e as Linhagens comerciais A e B. O manual da Ross AG 308 cita 3.496 gramas para lotes mistos aos 56 dias de idade. As 4 linhagens apresentaram PM inferior ao citado neste manual. Já o manual da Cobb 500 cita 3.308 gramas para lotes mistos, as quatro linhagens obtiveram desempenho superior ao que é citado no referido manual. O CR foi estatisticamente igual entre EMBRAPA 1 e 2 e Linhagem A (P>0,05), o CR da EMBRAPA 1 e 2 foi superior estatisticamente à linhagem B. As Linhagens A e B não diferiram estatisticamente sobre a mesma significância. $\mathrm{O}$ CR citado pelo manual Ross AG 308 é de 6.792 gramas de ração de 1 a 56 dias de idade. As quatro linhagens consumiram menos do que é citado por este manual. A CA não apresentou diferença estatística entre as linhagens da EMBRAPA 1 e 2 e a Linhagem A. A EMBRAPA 2 apresentou pior CA que a Linhagem B. As linhagens EMBRAPA 1, Linhagem A e B não apresentaram diferença estatística $(\mathrm{P}>0,05)$. O CA citado pelo manual da Ross AG 308 é 1,94. Apenas a linhagem EMBRAPA 2 apresentou consumo superior. EMBRAPA 1 e Linhagem A apresentaram exatamente esta CA, enquanto a Linhagem B valor ligeiramente inferior. O manual da Cobb 500 cita 2,05 de CA para lotes mistos para esta idade. Todas as quatro linhagens avaliadas apresentaram CA inferior numericamente. O GMD não diferiu entre as linhagens EMBRAPA 1 e a Linhagem A, como também não diferiu entre as linhagens EMBRAPA 2 , Linhagem A e B $(P>0,05)$. Houve diferença estatística entre a EMBRAPA 1, que foi superior à EMBRAPA 2 e a Linhagem B. O GMD citado pelo manual da Ross AG 308 é 61,68g. As quatro linhagens avaliadas apresentaram GMD menor, sendo a EMBRAPA 1 a que mais se aproximou deste valor. Não houve diferença estatística entre o PMT das quatro linhagens, no período de 1-56 dias. Com relação ao IEP, o da EMBRAPA 1 e Linhagem B foi superior à EMBRAPA 2, mas igual estatisticamente à Linhagem B. Não houve diferença estatística entre a EMBRAPA 2 e a Linhagem A.

A tabela 2 mostra a média do peso médio, consumo de ração, conversão alimentar, ganho médio diário de peso e índice de eficiência produtiva dos machos e fêmeas das quatro linhagens avaliadas.Os machos apresentaram melhor desempenho em PM, CR, CA, GMD e IEP em relação às fêmeas. O manual da Cobb 500 cita 3.737 gramas de PM para machos e 2.879 gramas para fêmeas. Os machos da EMBRAPA 1 foram os que apresentaram PM

Tabela 1 - Peso médio (PM), Consumo de Ração (CR), Conversão Alimentar (CA), Ganho Médio Diário de Peso (GMD), Percentual de Mortalidade (PMT) e IEP (Índice de Eficiência Produtiva) no período de 1-56 dias de idade

\begin{tabular}{lcccccc}
\hline Linhagens & \multirow{2}{*}{ PM(g)* } & CR $(\mathrm{g})^{*}$ & $\mathrm{CA}^{*}$ & GMD(g)* & PMT (\%) & IEP* \\
\hline EMBRAPA 1 & $3396 \mathrm{a}$ & $6620 \mathrm{a}$ & $111,94 \mathrm{ab}$ & $60,04 \mathrm{a}$ & 2,90 & $307 \mathrm{a}$ \\
\hline EMBRAPA 2 & $3316 \mathrm{~b}$ & $6680 \mathrm{a}$ & $2,01 \mathrm{a}$ & $58,53 \mathrm{~b}$ & 1,87 & $286 \mathrm{~b}$ \\
\hline Linhagem A & $3357 \mathrm{ab}$ & $6529 \mathrm{ab}$ & $1,94 \mathrm{ab}$ & $59,28 \mathrm{ab}$ & 1,87 & $300 \mathrm{ab}$ \\
\hline Linhagem B & $3324 \mathrm{~b}$ & $6284 \mathrm{~b}$ & $1,89 \mathrm{~b}$ & $58,67 \mathrm{~b}$ & 1,25 & $302 \mathrm{a}$ \\
\hline $\mathrm{CV}(\%)$ & 1,68 & 3,94 & 3,74 & 1,72 & 31,88 & 4,27 \\
\hline
\end{tabular}

*significativo $(\mathrm{P}<0,05)$;** altamente significativo $(\mathrm{P}<0,01)$; Médias seguidas de letras iguais nas colunas não diferem entre si $(\mathrm{P}>0,01)$ pelo teste de Tukey. 
Tabela 2 - Peso Médio (PM), Consumo de Ração (CR), Conversão Alimentar (CA), Ganho Médio Diário (GMD), no período de 1-56 dias de idade dos machos e das fêmeas

\begin{tabular}{|c|c|c|c|c|c|}
\hline \multicolumn{6}{|c|}{ Machos } \\
\hline Linhagens & PM (kg) & $\mathrm{CR}(\mathrm{kg})$ & $\mathrm{CA}$ & $\mathrm{GMD}(\mathrm{g})$ & IEP \\
\hline EMBRAPA 1 & 3734 & 6,81 & 1,84 & 65,96 & 356,40 \\
\hline EMBRAPA 2 & 3674 & 7,05 & 1,94 & 64,94 & 332,42 \\
\hline Linhagem A & 3682 & 6,90 & 1,89 & 65,07 & 338,99 \\
\hline Linhagem B & 3668 & 6,69 & 1,84 & 64,80 & 343,60 \\
\hline \multicolumn{6}{|c|}{ Fêmeas } \\
\hline Linhagens & PM (kg) & $\mathrm{CR}(\mathrm{kg})$ & $\mathrm{CA}$ & $\mathrm{GMD}(\mathrm{g})$ & IEP \\
\hline EMBRAPA 1 & 3,05 & 6,41 & 2,12 & 54,12 & 257,60 \\
\hline EMBRAPA 2 & 2,95 & 6,30 & 2,16 & 52,12 & 240,63 \\
\hline Linhagem A & 3,03 & 6,14 & 2,05 & 53,49 & 261,18 \\
\hline Linhagem B & 2,98 & 5,86 & 1,98 & 52,53 & 260,78 \\
\hline \multicolumn{6}{|c|}{ Machos x FêmeaS } \\
\hline Linhagens & PM (kg) & $\mathrm{CR}(\mathrm{kg})$ & $\mathrm{CA}$ & $\mathrm{GMD}(\mathrm{g})$ & IEP \\
\hline Machos & 3689 a & $6,86 \mathrm{a}$ & $1,86 \mathrm{a}$ & $65,19 \mathrm{a}$ & $342,85 \mathrm{a}$ \\
\hline Fêmeas & $3007 \mathrm{~b}$ & $6,37 \mathrm{~b}$ & $2,05 \mathrm{~b}$ & $53,07 \mathrm{~b}$ & $255,04 \mathrm{~b}$ \\
\hline Probabilidade & 0,01 & 0,01 & 0,01 & 0,01 & 0,01 \\
\hline
\end{tabular}

mais próximo ao que é citado no manual, todos porém apresentaram PM inferior. $O$ inverso aconteceu em relação às fêmeas onde todas apresentaram PM superior ao que é citado no referido manual. Porém todas a quatro linhagens apresentaram desempenho inferior ao citado no manual da Ross AG 308 que é de 3.078 gramas. O CR citado no referido manual para machos é 7.394 gramas. Todas as linhagens apresentaram menor CR para machos, enquanto que, para fêmeas, o manual cita 6.176 gramas, onde as fêmeas das linhagens da EMBRAPA apresentaram consumo superior e as fêmeas das linhagens comerciais consumo inferior. A CA citada no manual da Ross AG 308 para machos é 1,89 e para fêmeas 2,01 , o que ficou muito próximo aos valores obtidos nesse experimento. O manual da Cobb 500 cita 1,97 para machos e 2,16 para fêmeas, também muito semelhantes aos valores encontrados neste trabalho. O GMD, citado no manual da Ross AG 308, é 69,13 gramas, valor superior às linhagens testadas, sendo as linhagens EMBRAPA 1 e Linhagem A as que mais se aproximaram deste valor. Já para fêmeas, o mesmo manual cita o GMD de 54,21 gramas. Novamente as Linhagens EMBRAPA 1 e Linhagem A foram as que mais se aproximaram deste ganho médio diário de peso de 1-56 dias de idade, porém apresentando desempenho ligeiramente inferior ao que é indicado pelo manual.

\section{CONCLUSÕES}

Nas condições em que foi conduzido o experimento, conclui-se que o desempenho zootécnico dos frangos de corte das duas linhagens da EMBRAPA está satisfatório comparado ao das duas linhagens comerciais, pois apresentaram peso médio, consumo de ração, ganho médio de peso e IEP semelhantes aos 56 dias de idade.

Estudos econômicos são necessários para melhores conclusões sobre o desempenho das linhagens, envolvendo o desempenho produtivo das matrizes.

\section{REFERÊNCIAS BIBLIOGRÁFICAS}

AGROCERES. Manual de manejo de frangos Agross: objetivos de desempenho Ag 308. São Paulo : Agroceres Ross MG de Aves, 2000. 104p.

CAMPOS, E.J. Avicultura razões, fatos e divergências. Belo Horizonte : FEP-MVZ, 2000. 311p.

COBB. Manual de manejo de frangos Cobb 500: guia de manejo. São Paulo : Cobb-Vantress Brasil, 2001. 47p.

NATIONAL RESERCH COUNCIL, NRC. Nutrient requirements of poultry. 9.ed. Washington DC, 1994. 155p.

SAS PROGRAM. User guide for personal computer, Cary, North Caroline, 1997.

TALAMINI, D.; SANTOS FILHO, J. A avicultura de corte em 2002. Anuário 2003 da Avicultura Industrial. Porto Feliz, v.94, n.1107, p.14-18. 2003

Ciência Rural, v.35, n.2, mar-abr, 2005. 